\title{
Structures of cyanobacterial bicarbonate transporter SbtA and its complex with PII-like SbtB
}

\author{
Xiao-Yu Liu', Wen-Tao Hou', Liang Wang ${ }^{1}$, Bo Li', Yu Chen', Yuxing Chen', Yong-Liang Jiang ${ }^{1}$ and \\ Cong-Zhao Zhou (1)
}

Dear Editor,

Carbon and nitrogen, the uptake and intracellular metabolisms of which are tightly coupled ${ }^{1}$, are the two most fundamental nutrients for all living organisms. As one of the most ancient autotrophic bacteria, cyanobacteria utilize photosynthesis to convert the inorganic carbon $\left(C_{\mathrm{i}}\right)$ into carbohydrates. Carbon fixation is catalyzed by ribulose-1,5-bisphosphate carboxylase/oxygenase $(\mathrm{RuBisCO})$, which is a naturally inefficient enzyme ${ }^{2}$. In response to gradually decreased $\mathrm{CO}_{2}$ and elevated $\mathrm{O}_{2}$ levels in the atmosphere, cyanobacteria have evolved a unique $\mathrm{CO}_{2}$-concentrating mechanism (CCM), which can substantially accumulate $\mathrm{CO}_{2}$ in the vicinity of RuBisCO for improved carboxylation efficiency ${ }^{3}$. The cyanobacterial CCM consists of a subcellular self-assembled icosahedral microcompartment, termed the carboxysome, and several $C_{i}$ uptake systems ${ }^{3}$. To date, five $C_{i}$-uptake systems have been identified in cyanobacteria, including three bicarbonate transporters BicA, SbtA, and BCT1, in addition to two $\mathrm{CO}_{2}$-uptake complexes: $\mathrm{NDH}-\mathrm{I}_{3}$ and $\mathrm{NDH}-\mathrm{I}_{4}$.

SbtA, which is ubiquitous in cyanobacteria, is an inducible high-affinity sodium-dependent $\mathrm{HCO}_{3}{ }^{-}$symporter within the TC.2.A.83 family of $\mathrm{Na}^{+}$/solute symporters ${ }^{4,5}$. At a low level of intracellular $\mathrm{C}_{\mathrm{i}}$, SbtA of an up-regulated level can recruit its partner protein SbtB to the membrane $^{6}$. The $s b t B$ gene from the $s b t A-s b t B$ operon encodes a PII-like signaling protein that senses the intracellular level of the secondary messenger cAMP that correlates with high $C_{i}$ conditions, versus AMP at low $C_{i}$

\footnotetext{
Correspondence: Yuxing Chen (cyxing@ustc.edu.cn) or YongLiang Jiang (jyl@ustc.edu.cn) or Cong-Zhao Zhou (zcz@ustc.edu.cn)

${ }^{1}$ Hefei National Laboratory for Physical Sciences at the Microscale and School of Life Sciences, University of Science and Technology of China, Hefei, Anhui, China
}

conditions, thus functioning as a $\mathrm{C}_{\mathrm{i}}$ sensor in cyanobacteria ${ }^{6}$.

Here we purified Synechocystis sp. PCC 6803 SbtA and solved its cryo-EM structure at $3.50 \AA$ resolution (Supplementary Fig. S1). SbtA adopts a trimeric structure, each subunit of which consists of two inverted structural repeats, namely TM1-5 and TM6-10 (Fig. 1a), which are similar to each other in topology but have antiparallel orientations within the membrane (Supplementary Fig. S2). The two structural repeats possess a root-mean-square deviation (RMSD) of $2.63 \AA$ over $135 \mathrm{C} \alpha$ atoms. At the 3D level, all 10 TMs of each SbtA subunit are folded into two domains, a core domain of six TMs (TM3-5 and TM8-10) and a gate domain of four TMs (TM1-2 and TM6-7) (Fig. 1a; Supplementary Fig. S3). The two domains have a buried interface of $\sim 2000 \AA^{2}$, which is mainly mediated by hydrophobic interactions between residues from TM4-5 to TM9-10 of the core domain and the four TMs of the gate domain (Fig. 1a). Notably, the TM4 and TM9 helices in the core domain are unfolded and form a crossover at the middle. In the gate domain, TM1 and TM6 are divided into two helical moieties, forming a kink at the middle (Fig. 1a; Supplementary Fig. S3). In fact, this feature of discontinuous transmembrane helices is common in previously reported secondary active transporters ${ }^{7}$. Structural analysis showed that SbtA of a $5+5$ fold is topologically similar to Neisseria meningitides $\mathrm{ASBT}^{8}$. However, SbtA possesses distinct TM conformations from ASBT, especially those at the gate domain, with an RMSD of $4.4 \AA$ over 249 C $\alpha$ atoms.

The tightly packed trimeric structure of SbtA is exclusively stabilized by the gate domains at the center, with a threefold axis perpendicular to the membrane plane (Fig. 1b). The trefoil-like helical bundle of the gate domains possesses a completely buried interface area of $\sim 3000 \AA^{2}$, mainly via hydrophobic interactions. As 


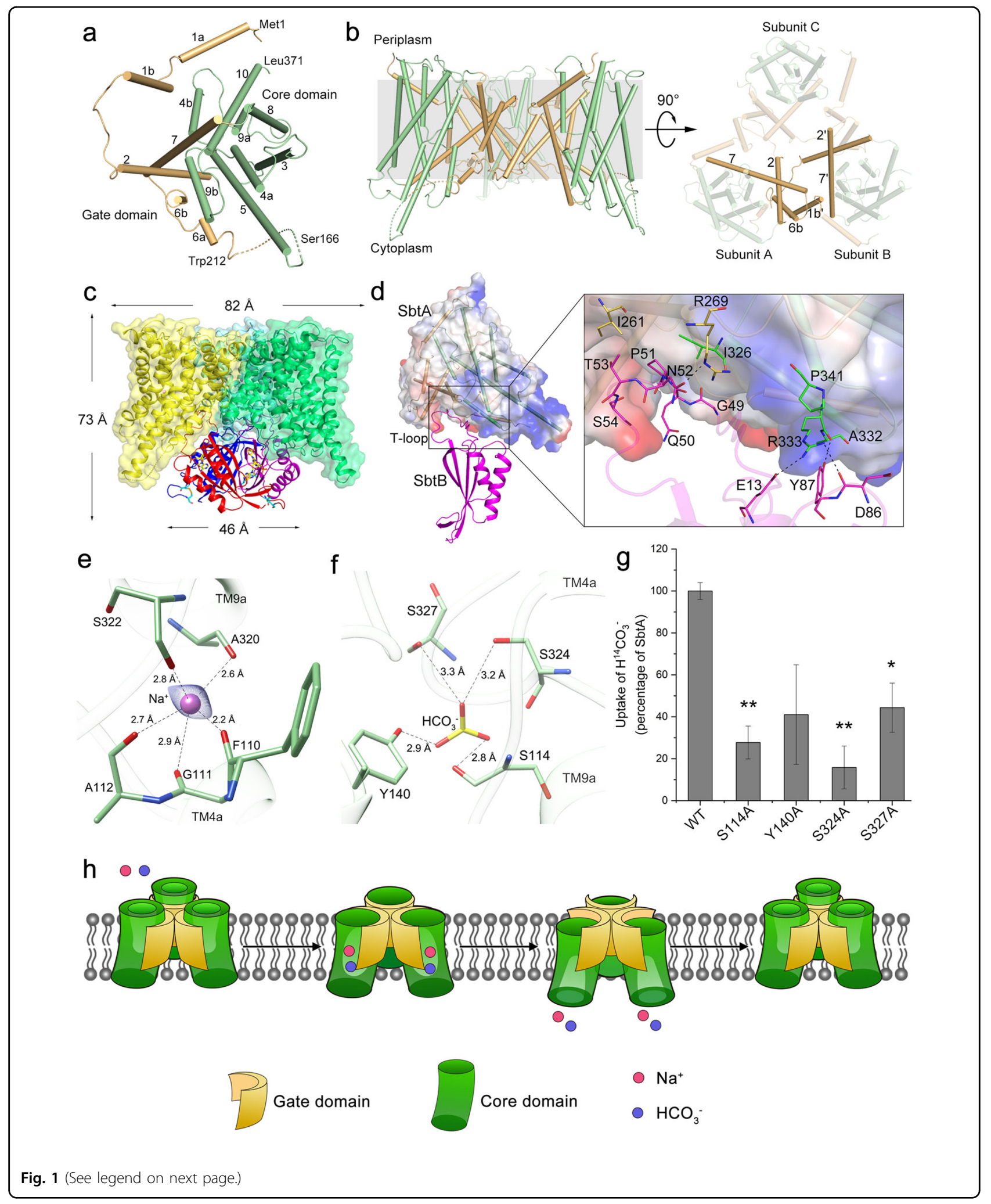


(see figure on previous page)

Fig. 1 Cryo-EM structures of SbtA and its complex with SbtB. a Overall structure of a SbtA subunit viewed from the extracellular side with helices shown as cylinders. $\mathbf{b}$ Side view of the trimeric structure of SbtA (left) and top view from the intracellular side (right). The gate domains are colored in orange, whereas the core domains are colored in green. The missing residues between Ser166 and Trp212 are indicated as a dashed line. c Cartoon representation of SbtA-SbtB complex structure. Three subunits of SbtB are colored in magentas, red and blue, respectively. The C-terminal disulfide bond between Cys105 and Cys110 is indicated by blue sticks. An AMP molecule in the nucleotide-binding cleft is shown as yellow sticks. $\mathbf{d}$ Interfaces between a pair of SbtA and SbtB subunits. The interacting residues are shown as sticks, with the polar interactions indicated by dashed lines. e The putative $\mathrm{Na}^{+}$-binding site. The $\mathrm{Na}^{+}$is shown as a violet sphere whereas the $\mathrm{Na}^{+}$-binding residues are shown as sticks. The polar interactions are indicated by dashed lines. The cryo-EM density map of $\mathrm{Na}^{+}$is shown in blue mesh. $\mathbf{f}$ The putative $\mathrm{HCO}_{3}{ }^{-}$-binding site modeled by $\mathrm{HADDOCK}$. The $\mathrm{HCO}_{3}{ }^{-}$molecule is shown as sticks and colored by atoms. $\mathbf{g}$ The $\mathrm{HCO}_{3}{ }^{-}$transport activity assays of the wild-type SbtA and mutants in E. coli membrane vesicles. Three independent experiments were performed for each assay. The means and standard deviations were calculated and the data are presented as means \pm SD. Two-tailed Student's $t$-test is used for the comparison of statistical significance. The $P$ values of $<0.05$ and $<0.01$ are indicated with * and **, respectively. $\mathbf{h ~ A}$ proposed elevator mechanism of $\mathrm{Na}^{+}$-dependent bicarbonate transport of the trimeric SbtA. The $\mathrm{Na}^{+}$ and $\mathrm{HCO}_{3}{ }^{-}$are shown as red and blue spheres, respectively. SbtA of an outward-open conformation recruits the substrates $\mathrm{HCO}_{3}{ }^{-}$and $\mathrm{Na}^{+}$from the periplasm, accompanied by a rigid-body movement of core domains (green) against the immobile gate domains (yellow). Afterwards, the substrates are released into the cytosol followed by the turnover of SbtA into the resting state.

observable from the extracellular side, the N-terminal moieties of three TM7 helices are bent and interact with each other, whereas from the intracellular side, the Ntermini of three TM2 helices form pairwise crossovers via hydrophobic interactions (Fig. 1b). In addition, TM1b of one subunit is positioned against TM6b of the neighboring subunit via hydrophobic interactions, further stabilizing the trimeric structure at the lateral side (Fig. 1b).

It has been reported that when SbtB and SbtA are coexpressed in Escherichia coli (E. coli), they form a complex $^{9}$. To elucidate the fine interaction mode between SbtA and SbtB, we purified the SbtA-SbtB complex in the presence of AMP and solved its $3.15 \AA$ cryo-EM structure (Supplementary Fig. S4). The SbtB trimer is associated with the intracellular face of the SbtA trimer (Fig. 1c), sharing an overlapped threefold axis. The overall shape of the complex resembles a cylindrical cone of $\sim 73 \AA$ in height, and $\sim 82$ and $46 \AA$ in diameter for the SbtA and SbtB trimers, respectively (Fig. 1c). Similar to the previously reported PII and PII-like structures ${ }^{10}$, SbtB also adopts a trimeric structure, each subunit of which shows a canonical PII ferredoxin-like fold with a well-structured T-loop (Fig. 1c; Supplementary Fig. S5). The C-terminal CGPxGC motif, which is conserved in some SbtB homologs, also forms a hairpin structure via a disulfide bond between Cys105 and Cys110 (Fig. 1c; Supplementary Fig. S5). In addition, an AMP molecule binds to the nucleotide-binding cleft between each pair of neighboring SbtB subunits (Fig. 1c; Supplementary Fig. S5).

In the complex, the core structure of SbtB does not directly interact with the intracellular surface of SbtA. Instead, the structured T-loop of SbtB, which extends $\sim 20 \AA$ from the core structure of SbtB, is inserted into the inter-domain cleft of the corresponding SbtA subunit (Fig. 1d), yielding an interface area of $\sim 740 \AA^{2}$. The interdomain cleft of SbtA is formed by the intracellular parts of TM2 and TM7 of the gate domain, in addition to TM9 and TM10 of the core domain. The extended SbtB T-loop runs along the hydrophobic inter-domain cleft of SbtA, forming extensive hydrophobic interactions (Fig. 1d) via relatively conserved residues (Supplementary Fig. S6). Similar to the complex structure of AmtB-GlnK, in which the T-loop of PII protein GlnK inserts deeply into the cytoplasmic pore exit of the ammonia channel $A m \mathrm{BB}^{11}$, the SbtA-SbtB structure showed that SbtB T-loop partially blocks the substrate tunnel exit of SbtA, indicating that SbtB might attenuate the transport activity of SbtA under certain physiological conditions. Consistently, the previous results showed that SbtB inhibited the SbtAmediated $\mathrm{HCO}_{3}{ }^{-}$uptake in E. coli ${ }^{9}$. However, a recent report demonstrated that the light-regulated SbtA function in vivo is independent of SbtB in Synechococcus PCC $7942^{12}$. Thus the fine in vivo regulatory mechanism of SbtA-SbtB remains unsolved, but might be involved in the coordinated regulation of light-dark transitions, the intracellular homeostasis of adenyl-nucleotides and redox status.

At the peripheral side of TM crossover within the center of SbtA core domain in the SbtA-SbtB complex, there is a clear cryo-EM density most likely corresponding to a sodium ion (Fig. 1e), given SbtA is a $\mathrm{Na}^{+}$-dependent transporter. In each subunit, the $\mathrm{Na}^{+}$forms five coordinate bonds with the main-chain oxygens of residues Phe110, Gly111, and Ala112 from TM4a and Ala320 and Ser322 from TM9a (Fig. 1e). Notably, in the previously reported $\mathrm{Na}^{+}$-dependent transporters ${ }^{8,13}, \mathrm{Na}^{+}$and its cotransported substrate are located nearby the crossover of TMs. We thus docked $\mathrm{HCO}_{3}^{-}$in the vicinity of the crossover of the complexed SbtA via HADDOCK ${ }^{14}$. In this docking model, the $\mathrm{HCO}_{3}{ }^{-}$-binding site is located at the interface of the core and gate domains. It binds to a cavity formed by the other side of the crossover and forms four hydrogen bonds with Ser114, Tyr140, Ser324, and Ser327 (Fig. 1f). Sequence analysis revealed that most of these putative substrate-binding residues are conserved among SbtA homologs (Supplementary Fig. S7). 
Furthermore, mutating any of these putative $\mathrm{HCO}_{3}{ }^{-}$binding residues to Ala led to a dramatic decrease of $\mathrm{HCO}_{3}{ }^{-}$transport activity of SbtA in E. coli membrane vesicles to about $10 \%-40 \%$ of the wild-type level (Fig. 1g). The results demonstrated that these residues are necessary for optimal transport activity of SbtA.

Structural analysis revealed that the substrate-binding sites of free SbtA are inaccessible to the solvent from either intracellular or extracellular side. Thus, the structure of free SbtA represents an occluded conformation. Respectively superimposing the two individual domains of each SbtA subunit against those in the SbtB-bound form showed little structural variations (Supplementary Fig. S8a). However, upon binding to SbtB, the two domains of each SbtA subunit undergo a significant rigid-body movement against each other. Given the superimposed gate domains, each core domain of the complexed SbtA tilts about $10^{\circ}$ against the trefoil-like center, accompanied by a $4-\AA$ slide of the core domain toward the intracellular space (Supplementary Fig. S8b). As a result, the two domains of SbtA in SbtB-bound form are relatively separated from each other, yielding an inward-open conformation (Supplementary Fig. S8b). The present structures of SbtA in the free and SbtB-bound forms capture two snapshots in the transport cycle which enable us to propose a putative model for SbtA-mediated $\mathrm{HCO}_{3}{ }^{-}$ transport (Fig. 1h). Initially, SbtA was assumed to adopt an outward-open conformation ready to uptake the substrates $\mathrm{HCO}_{3}{ }^{-}$and $\mathrm{Na}^{+}$. Substrate binding most likely promotes SbtA to adopt an occluded conformation, as seen in our free SbtA structure with a $\mathrm{Na}^{+}$bound in the pocket. Afterwards, the core domains of SbtA slide toward the cytosolic side against the immobile gate domains, resulting in an inward-open conformation of SbtA. Accordingly, the substrate tunnel is open toward the intracellular space (Supplementary Fig. S8b). Finally, the substrates are released to the cytosol, accompanied by the turnover of SbtA to the resting state for the next cycle of transport. This transport model of SbtA is reminiscent of the elevator alternating-access transport mechanism, in which the substrate-binding domain moves along the relatively immobile scaffold domain ${ }^{15}$.

In summary, this study provides structural insights into the cyanobacterial high-affinity $\mathrm{HCO}_{3}{ }^{-}$transporter SbtA, which exhibits a new architecture of $\mathrm{Na}^{+}$/solute symporters and its fine interaction pattern with a PII-like protein SbtB. Structural analysis combined with sitedirected mutagenesis enabled us to identify the substratebinding pocket and propose an elevator transport mechanism of SbtA.

\section{Acknowledgements}

We thank Dr. Yong-Xiang Gao for technical support on cryo-EM data collection of SbtA at the Center for Integrative Imaging of University of Science and Technology of China (USTC). We thank Dr. Li-Hong Chen for technical support on cryo-EM data collection of SbtA-SbtB complex at the Center for Biological Imaging at the Institute of Biophysics (IBP), Chinese Academy of Sciences. We thank Mr. Jishu Ren (USTC) for the assistance on the transport assays. This research was supported by the Strategic Priority Research Program of the Chinese Academy of Sciences (XDB37020301 and XDA24020302), the National Natural Science Foundation of China (31630001 and 31621002), and the Ministry of Science and Technology of China (2016YFA0400900). Y.-L.J. thanks the Youth Innovation Promotion Association of Chinese Academy of Sciences for their support (Membership No. 2020452).

\section{Author contributions}

C.-Z.Z., Y.-L.J. and Y.C. conceived the project. X.-Y.L. and B.L. performed molecular cloning. X.-Y.L. performed protein purification and cryo-EM sample preparation. Y.-L.J., L.W. and Y.C. conducted cryo-EM data collection, structure determination and model building. W.-T.H. and X.-Y.L. performed transport assays. All authors analyzed the data and contributed to the paper preparation. Y.-L.J., X.-Y.L. and C.-Z.Z. wrote the paper.

\section{Data availability}

The cryo-EM structures of SbtA and SbtA-SbtB have been deposited at PDB under the codes of 7CYE and 7CYF, respectively. The cryo-EM density maps of SbtA and SbtA-SbtB have been deposited at the Electron Microscopy Data Bank (EMD-30498 for SbtA and EMD-30499 for SbtA-SbtB).

Conflict of interest

The authors declare no competing interests.

\section{Publisher's note}

Springer Nature remains neutral with regard to jurisdictional claims in published maps and institutional affiliations.

Supplementary information The online version contains supplementary material available at https://doi.org/10.1038/s41421-021-00287-w.

Received: 20 April 2021 Revised: 20 April 2021 Accepted: 3 June 2021 Published online: 10 August 2021

\section{References}

1. Zhang, C. C., Zhou, C. Z., Burnap, R. L. \& Peng, L. Carbon/Nitrogen Metabolic Balance: lessons from Cyanobacteria. Trends Plant Sci. 23, 1116-1130 (2018).

2. Bracher, A., Whitney, S. M., Hartl, F. U. \& Hayer-Hartl, M. Biogenesis and Metabolic Maintenance of Rubisco. Annu. Rev. Plant Biol. 68, 29-60 (2017).

3. Price, G. D., Badger, M. R., Woodger, F. J. \& Long, B. M. Advances in understanding the cyanobacterial $\mathrm{CO}_{2}$-concentrating-mechanism (CCM): functional components, $\mathrm{Ci}$ transporters, diversity, genetic regulation and prospects for engineering into plants. J. Exp. Bot. 59, 1441-1461 (2008).

4. Shibata, M. et al. Genes essential to sodium-dependent bicarbonate transport in cyanobacteria: function and phylogenetic analysis. J. Biol. Chem. 277, 18658-18664 (2002).

5. Price, G. D., Shelden, M. C. \& Howitt, S. M. Membrane topology of the cyanobacterial bicarbonate transporter, SbtA, and identification of potential regulatory loops. Mol. Membr. Biol. 28, 265-275 (2011).

6. Selim, K. A., Haase, F., Hartmann, M. D., Hagemann, M. \& Forchhammer, K. PIIlike signaling protein SbtB links CAMP sensing with cyanobacterial inorganic carbon response. Proc. Natl. Acad. Sci. USA 115, E4861-E4869 (2018).

7. Screpanti, E. \& Hunte, C. Discontinuous membrane helices in transport proteins and their correlation with function. J. Struct. Biol. 159, 261-267 (2007).

8. Hu, N. J., Iwata, S., Cameron, A. D. \& Drew, D. Crystal structure of a bacterial homologue of the bile acid sodium symporter ASBT. Nature 478, 408-411 (2011).

9. Du, J., Forster, B., Rourke, L., Howitt, S. M. \& Price, G. D. Characterisation of cyanobacterial bicarbonate transporters in E. coli shows that SbtA homologs are functional in this heterologous expression system. PLOS ONE 9, e115905 (2014).

10. Forchhammer, K. \& Luddecke, J. Sensory properties of the PII signalling protein family. FEBS J. 283, 425-437 (2016). 
11. Conroy, M. J. et al. The crystal structure of the Escherichia coli AmtB-GInK complex reveals how GlnK regulates the ammonia channel. Proc. Natl. Acad. Sci. USA 104, 1213-1218 (2007).

12. Förster, B. et al. Regulatory adenylnucleotide-mediated binding of the Pll-like protein SbtB to the cyanobacterial bicarbonate transporter SbtA is controlled by the cellular energy state. bioRxiv https://doi.org/10.1101/2021.02.14.431189 (2021).
13. Wang, C. et al. Structural mechanism of the active bicarbonate transporter from cyanobacteria. Nat. Plants 5, 1184-1193 (2019).

14. de Vries, S. J., van Dijk, M. \& Bonvin, A. M. The HADDOCK web server for datadriven biomolecular docking. Nat. Protoc. 5, 883-897 (2010).

15. Drew, D. \& Boudker, O. Shared Molecular Mechanisms of Membrane Transporters. Annu. Rev. Biochem. 85, 543-572 (2016). 